\title{
Single dietary amino acids control resting egg production and affect population growth of a key freshwater herbivore
}

\author{
Ulrike Koch · Dominik Martin-Creuzburg • \\ Hans-Peter Grossart • Dietmar Straile
}

\begin{abstract}
The enormous success of the genus Daphnia in freshwater ecosystems is at least partially due to their cyclical parthenogenetic life cycle, in which asexual and sexual reproduction alternate periodically. This temporal change between reproductive strategies allows for (1) rapid population growth via subitaneously developing eggs when environmental conditions are appropriate and (2) the maintenance of genetic diversity via sexual reproduction and the production of resting eggs when environmental conditions deteriorate. We show here that dietary amino acids are involved in triggering the switch between reproductive modes in Daphnia pulex. Supplementation experiments demonstrate that specific dietary amino acids, in particular arginine and histidine, avert crowding-induced resting egg production, enhance subitaneous reproduction by increasing algal food quality and, as a combined effect of both processes, increase population growth rates. These findings suggest that the availability of single dietary amino acids potentially affects the seasonal dynamics and long-term persistence of Daphnia populations in the field, which may have consequences for the efficiency of carbon transfer and thus the trophic structure of freshwater food webs.
\end{abstract}

Communicated by Ulrich Sommer.

U. Koch (凶) · D. Martin-Creuzburg · D. Straile

Limnological Institute, University of Konstanz, Mainaustrasse 252, 78464 Constance, Germany

e-mail: ulrike.koch76@gmx.de

\section{H.-P. Grossart}

Leibniz-Institute of Freshwater Ecology and Inland Fisheries Berlin, Limnology of Stratified Lakes, Alte Fischerhütte 2 ,

OT Neuglobsow, 16775 Stechlin, Germany
Keywords Daphnia - Food quality - Reproductive mode . Parthenogenesis $\cdot$ Resting eggs

\section{Introduction}

The elemental and biochemical composition of plants and algae significantly affects the performance of herbivores. For example, the mineral content and primary and secondary metabolite contents of plants and algae can influence herbivore foraging behaviour (Berner et al. 2005; Schatz and McCauley 2007), somatic growth and reproduction (Sterner and Elser 2002; Von Elert 2002; MartinCreuzburg et al. 2009), life history strategy (Twombly et al. 1998), immune function (Bukovinszky et al. 2009; Smilanich et al. 2009), anti-predator defence (Kazana et al. 2007) and genetic diversity (Weider et al. 2008). As a consequence, the elemental and biochemical composition of herbal food sources potentially affects the population dynamics of herbivores and thus the efficiency with which carbon and organic matter are transferred to higher trophic levels (Müller-Navarra et al. 2000; Wacker and Von Elert 2001; Sterner and Elser 2002).

Seasonal dynamics of the freshwater keystone herbivore Daphnia spp. (Crustacea, Anomopoda, Daphnidae) strongly affect the efficiency of carbon transfer from primary producers to higher trophic levels. This is due both to the high grazing impact daphnids impose upon phytoplankton and to the fact that daphnids are the principal prey of many invertebrate predators and planktivorous fish species. As a result, demographic changes in Daphnia spp. and its population dynamics can have profound effects on the trophic structure and function of freshwater food webs (Lampert 1987; Leibold 1989; Carpenter et al. 2001). The life cycle of daphnids is usually characterised by a cyclical 
parthenogenesis, i.e. asexual (parthenogenetic) reproduction alternates periodically with sexual reproduction. In the field, Daphnia populations consist primarily of parthenogenetic females reproducing via subitaneously developing eggs. Sexual reproduction, which in Daphnia is associated with the production of resting stages (so-called ephippia, containing up to two diapausing eggs), is induced by deteriorating environmental conditions and often restricted to specific times of the year, such as during short periods in early summer and late autumn (Cáceres 1998; Jankowski and Straile 2004). This temporal change between reproductive strategies allows for both rapid population growth via parthenogenesis when environmental conditions are appropriate, thereby avoiding the costs of producing males (Innes et al. 2000), and the maintenance of genetic diversity via sexual reproduction and the production of resting eggs when environmental conditions deteriorate (Hairston 1996; Jankowski and Straile 2004).

Resting egg production is an important life history trait which enables survival during unfavourable environmental conditions, such as low temperature, intense predation, desiccation or starvation (Schwartz and Hebert 1987; Ślusarczyk 1995; Alekseev and Lampert 2001), and facilitates dispersal in time and space (Hairston and Kearns 2002; Panov et al. 2004; Louette and De Meester 2005). However, the production of resting eggs is supposed to be associated with higher costs compared to the production of subitaneously developing eggs because during each molt cycle, a single female can either produce one clutch of several subitaneously developing eggs or one ephippium containing only one or two resting eggs. This trade-off in quantity between subitaneously developing eggs and resting eggs, together with the costs associated with male production, presumably have favoured the evolution of an inducible rather than a fixed switch between reproductive modes. The inducibility of resting egg production in Daphnia has been extensively studied, and several environmental factors have been shown to induce the switch between reproductive modes, including population density, food concentration, photoperiod or fish kairomones (Stross and Hill 1965; Kleiven et al.1992; Ślusarczyk 1995, 2001).

In past decades, a considerable amount of research focused on the effects of food quality on the performance of Daphnia species and the associated consequences for food web processes. In particular, a low availability of dietary phosphorus (Sterner and Elser 2002), polyunsaturated fatty acids (PUFAs) (Müller-Navarra et al. 2000; Wacker and Von Elert 2001; Von Elert 2002) and sterols (Von Elert et al. 2003; Martin-Creuzburg et al. 2005, 2009) has been shown to impair the growth and reproduction of Daphnia species. However, up to now, food quality studies have focused almost exclusively on the parthenogenetic part of the Daphnia life cycle, thereby neglecting the effects of food quality on resting egg production. In fact, only one published study has focused on this aspect, documenting that food quality can have pronounced effects on the frequency of resting egg production (Abrusán et al. 2007).

In a previous publication, we demonstrated that dietary proteins are involved in triggering the switch between subitaneous reproduction and resting egg production in two Daphnia species, D. galeata and D. pulex. (Koch et al. 2009). This finding led us to suggest that the availability of dietary proteins may significantly affect long-term persistence and dispersal and, potentially, also the genetic diversity of Daphnia populations in the field. Moreover, our data revealed that protein supplements can improve parthenogenetic fecundity of the herbivore, which implies that a deficiency in dietary proteins is an important food quality constraint. In the study reported here, we investigated whether the observed effects are mediated by single amino acids rather than unspecific proteins. By supplementing the nutritionally mediocre green alga Scenedesmus obliquus, which has been shown to cause resting egg production in crowded daphnids (Koch et al. 2009), with ten presumably essential amino acids, we tested the potential of single amino acids to control the switch between reproductive modes in a clone of Daphnia pulex.

\section{Materials and methods}

\section{Cultivation of food organisms}

The green alga Scenedesmus obliquus (SAG 276-3a) and the cryptophyte Cryptomonas sp. (SAG 28.80) were cultured semi-continuously in aerated 5 -L vessels at a dilution rate of 0.25 day $^{-1}\left(20^{\circ} \mathrm{C}\right.$; illumination at $146 \mu \mathrm{mol}$ quanta $\mathrm{s}^{-1} \mathrm{~m}^{-2}$ ). S. obliquus was grown in 'Cyano' medium (Jüttner et al. 1983) and Cryptomonas sp. in WC medium supplemented with vitamins (Guillard 1975). Preliminary experiments revealed that the vitamins used to cultivate Cryptomonas sp. did not affect ephippia production in the D. pulex feeding on S. obliquus.

\section{Daphnia growth experiments}

Experiments were conducted with an obligate parthenogenetic $D$. pulex clone, which is characterised by a naturally high allocation to ephippia production. The low density at which ephippia production can be induced in this clone [35-50 individuals (ind.) $\mathrm{L}^{-1}$ ] minimises competition and interference among individuals in the experimental beakers. Besides crowding, all other known stimuli for resting egg production were excluded, i.e. food limitation, fish kairomones or changes in photoperiod. The 
pre-experimental daphnids were raised in filtered lake water (pore size of membrane filter $0.45 \mu \mathrm{m}$ ) with saturating concentrations of $S$. obliquus at constant densities and continuous dim light for at least two generations. Experiments were conducted with female third-brood juveniles born within $12 \mathrm{~h}$.

All experiments lasted 16 days, i.e. a time intervals which allowed for three subitaneous or two ephippial broods, and were run under continuous dim light at $20^{\circ} \mathrm{C}$ in glass beakers filled with $200 \mathrm{~mL}$ of filtered lake water (pore size $0.45 \mu \mathrm{m}$ ) containing saturating food concentrations $\left(2 \mathrm{mg} \mathrm{C} \mathrm{L}^{-1}\right)$. Each jar contained ten animals to stimulate crowding-induced ephippia production (four replicates per treatment). Animals were transferred each day into new beakers with freshly prepared food suspensions, and the number of released ephippia and neonates was determined. During the experiments, daphnids were able to produce at most three subitaneous or two ephippial broods, respectively. Ephippia were usually released 4 days after the previous reproduction, whereas subitaneous reproduction could occur every second day. Allocation to resting egg production was determined as the number of produced ephippia; allocation to subitaneous reproduction was determined as the cumulative number of produced neonates. Population growth rates $(r)$ were estimated iteratively using the Euler-Lotka equation:

$1=\sum_{x=0}^{n} l_{x} m_{x} \mathrm{e}^{-r x}$

wherein $l_{x}$ is the age-specific survivorship, $m_{x}$ is the agespecific fecundity (number of neonates per individual) and $x$ is the age at reproduction (in days). The probability of survival $\left(l_{x}\right)$ was estimated from the mortality that occurred during the experiments.

\section{Supplementation of amino acids}

Amino acids (AA) were dissolved in ultrapure water and stored at $-20^{\circ} \mathrm{C}$. Aliquots of these stock solutions were added to the experimental beakers containing $2 \mathrm{mg} \mathrm{C} \mathrm{L}^{-1}$ of $S$. obliquus. The ten amino acids [Essential L-Amino Acid kit LAA1 (Sigma, St. Louis, MO) containing arginine (arg), histidine (his), isoleucine (ile), leucine (leu), lysine (lys), methionine (met), phenylalanine (phe), threonine (thr), tryptophane (try), valine (val)] were supplemented separately or in combination. In a first set of experiments, a mixture of all ten amino acids was initally supplemented to the experimental beakers, resulting in a concentration of $25 \mu \mathrm{M}$ per amino acid in each beaker. Subsequently, each amino acid was provided in a concentration-dependent manner, i.e. $25,12.5,6.25,3.125$ and $0 \mu \mathrm{M}$ amino acid. In a second set of experiments, amino acids were tested separately at a concentration of $25 \mu \mathrm{M}$ amino acid. In addition, arginine and histidine were tested in different concentrations $(50,25,0 \mu \mathrm{M})$ either separately or in combination.

Amino acid analyses

For the analysis of particulate combined amino acids, approximately $1 \mathrm{mg}$ particulate organic carbon (POC) of Cryptomonas sp. or S. obliquus were concentrated on precombusted GF/F filters ( $25 \mathrm{~mm}$; Whatman, New York, $\mathrm{NY}$ ) and stored at $-80^{\circ} \mathrm{C}$. Prior to analysis the samples were hydrolysed with $6 \mathrm{M} \mathrm{HCl}$ at $155^{\circ} \mathrm{C}$ for $1 \mathrm{~h}$ and neutralised with $6 \mathrm{M} \mathrm{NaOH}$. Filtered aliquots were derivatised with $o$-phthaldialdehyde (OPA) and analysed in a high-performance liquid chromatography (HPLC) system (HP1000; Agilent Tech, Santa Clara, CA) equipped with a fluorescence detector (Grossart et al. 2007). With this method, arginine and citrulline could not be separated from each other. Particulate organic carbon, nitrogen and phosphorus were analysed as described in Martin-Creuzburg et al. (2009).

\section{Statistical analyses}

Cumulative numbers of produced ephippia and neonates were analysed using one-way analyses of variance (ANOVA) with post hoc comparisons (Tukey's HSD). Dose-dependent responses to amino acids were analysed using non-linear regressions: an exponentially declining function was fitted to the data to describe the functional response of ephippia production, and a modified Michaelis-Menten function with a non-zero $y$ intercept was fitted to describe the functional response of neonate production and population growth rate. Multiple regression analyses with amino acid supplementation and ephippia production as independent variables were used to disentangle the direct (improvement of food quality) and indirect (switch in reproductive mode) effects of amino acid supplementation on neonate production and population growth rate. Pairwise comparisons using one-way ANOVAs were used to compare single amino acid treatments with unsupplemented S. obliquus. Dose-response effects of arginine and histidine were analysed using analysis of covariance (ANCOVA).

\section{Results}

The green alga Scenedesmus obliquus and the flagellate Cryptomonas sp. caused different responses in crowded Daphnia pulex: animals fed with $S$. obliquus showed a high investment in resting egg production, whereas those fed 
with Cryptomonas sp. reproduced only subitaneously (Fig. 1). The molar carbon to nitrogen $(\mathrm{C}: \mathrm{N})$ and carbon to phosphorus (C:P) ratios of $S$. obliquus [mean \pm standard deviation (SD): C:N 5.4 \pm 0.1 ; C:P $86.8 \pm 15.7]$ and Cryptomonas sp. (C:N $5.6 \pm 0.1$; C:P $117.1 \pm 15.7$ ) were low, indicating a high nitrogen and phosphorus content of the food suspensions. Thus, a limitation of D. pulex by nitrogen or phosphorus is rather unlikely.

Amino acid analyses revealed that Cryptomonas sp. contained considerably higher amounts of each amino acid analysed in this study than $S$. obliquus (Table 1). Supplementation of a $S$. obliquus food suspension with a mixture of ten presumably essential amino acids ( $25 \mu \mathrm{M}$ each) inhibited ephippia production of $D$. pulex and accordingly increased the production of subitaneous offspring (Tukey's HSD, $p<0.05$; following ANOVA, $F_{2,9}=39.5$ for ephippia production, $F_{2,9}=32.8$ for subitaneous offspring, $p<0.001$; Fig. 1).

Ephippia production declined exponentially $(y=$ $\left.\mathrm{e}^{-0.58 x}, \quad F_{1,18}=53.5, \quad p<0.0001\right) \quad$ when increasing amounts of the amino acid mixture were added to the diet. Ephippia production was close to zero at a concentration of $6.25 \mu \mathrm{M}$ of each amino acid (Fig. 2a). Both neonate production $\{$ Fig. $2 \mathrm{a} ; \quad y=22.4+60 \times[x /(x+22.8)]$, $\left.F_{3,17}=22.3, p<0.0001\right\}$ and population growth rates \{Fig. $2 \mathrm{~b} ; y=0.23+0.11 \times[x /(x+3.1)], F_{3,17}=27.0$, $p<0.0001\}$ increased with increasing amino acid supplementation following a saturation function. Note that neonate production as well as population growth rates increased further after ephippia production had ceasedi.e. at an amino acid concentration of $>6.25 \mu \mathrm{M}$ (simple regression models for neonates: $R^{2}=0.68, F_{2,12}=21.33$,

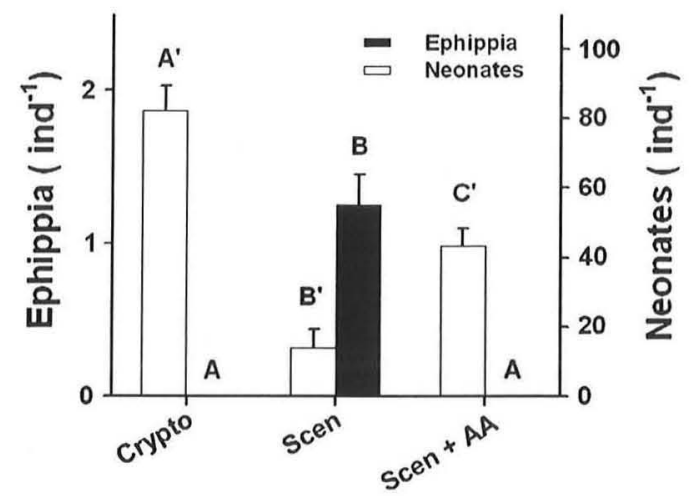

Fig. 1 Ephippia and neonate production of Daphnia pulex fed Cryptomonas sp. (Crypto), Scenedesmus obliquus (Scen), or S. obliquus supplemented with a mixture of ten amino acids $($ Scen $+A A)$ in a concentration of $25 \mu \mathrm{M} \mathrm{AA}^{-1}$. Data are presented as the cumulative numbers of ephippia and neonates produced per individual within 16 days. Error bars Mean + standard error (SE). Significant differences are labeled with different letters based on Tukey's HSD at $p<0.05$ following analysis of variance (ANOVA): $A, B, C$ Ephippia production, $A^{\prime}, B^{\prime}, C^{\prime}$ neonate production $p<0.001$; for population growth rate: $R^{2}=0.48$, $F_{2,12}=9.28, p<0.02$ ). A multiple regression model indicated that for the full range of amino acid supplementations, neonate production (no. ind ${ }^{-1}$ ) and population growth rates $r\left(\mathrm{day}^{-1}\right)$ increased with declining ephippia production (no. ind ${ }^{-1}$ ) and increasing amino acid supplementation $\left(\mu \mathrm{M} \mathrm{AA}^{-1}\right)$ :

1. neonates $=31.2-11.2 \times$ ephippia $+0.9 \times \mathrm{AA}$; $\left(R^{2}=0.82, F_{3,17}=38, p<0.0001\right)$

2. $r=0.3-0.07$ ephippia $+0.001 \times \mathrm{AA} ; \quad\left(R^{2}=0.94\right.$, $\left.F_{3,17}=149, p<0.0001\right)$.

Note that both ephippia production and amino acid supplementation contributed significantly to both models (all $p<0.005$ ).

The experiments involving supplementation of a $S$. obliquus food suspension with a single amino acid at a concentration of $25 \mu \mathrm{M}$ indicated that arginine and histidine were particularly effective in reducing ephippia production and increasing neonate numbers (Fig. 3). Pairwise comparisons using one-way ANOVAs between the two amino acid treatments and $S$. obliquus without amino acid addition revealed significant differences (arginine: $F_{1,6}=$ $6.5, p<0.05$ for ephippia production, $F_{1,6}=7.6, p<0.05$ for subitaneous neonate production; histidine: $F_{1,6}=11.8$, $p<0.05$ for ephippia production, $F_{1,6}=15.9, p<0.05$ for subitaneous neonate production).

Increasing supplementation with arginine and histidine (either separately or in combination) decreased ephippia production and accordingly increased neonate production and therewith population growth rate (Fig. 4). The ANCOVA with the supplemented concentration (25 or $50 \mu \mathrm{M}$ ) as a covariate and the specific amino acid (arginine, histidine, or arginine and histidine) as a categorical factor revealed a significant influence of the supplemented amino acid concentration as well as significant differences between treatments (Table 2). However, no significant interaction effects (all $p>0.1$ ) were detected for ephippia and neonate production or for population growth rate. Tukey's HSD suggested that histidine was less efficient in reducing ephippia production than arginine or a combination of arginine and histidine $(p<0.05)$. In contrast, the combined addition of arginine and histidine yielded a higher neonate production and higher population growth rates than the addition of arginine and histidine individually $(p<0.05)$.

\section{Discussion}

The results of our study demonstrate that specific dietary amino acids suppress resting egg production and 
Table 1 Content of selected amino acids in Cryptomonas sp. and Scenedesmus obliquus
Data are expressed as the mean

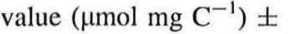

standard deviation $(n=3)$

a The abbreviations of the amino acids are given in the "Materials and methods"

b His, Arg, Thr, Met, Val, Phe, Ile and Leu were assumed to be essential and used in the supplementation experiments; lysine was not measured

\begin{tabular}{lcl}
\hline Amino acids analysed $^{\mathrm{a}}$ & $\begin{array}{l}\text { Cryptomonas sp. } \\
\left(\mu \mathrm{mol} \mathrm{mg} \mathrm{C}^{-1}\right)\end{array}$ & $\begin{array}{l}\text { Scenedesmus obliquus } \\
\left(\mu \mathrm{mol} \mathrm{mg} \mathrm{C}^{-1}\right)\end{array}$ \\
\hline Essential amino acids & \\
His & $0.574 \pm 0.072$ & $0.211 \pm 0.021$ \\
Arg/Cit & $0.709 \pm 0.087$ & $0.252 \pm 0.021$ \\
Thr & $0.711 \pm 0.095$ & $0.306 \pm 0.028$ \\
Met & $0.641 \pm 0.113$ & $0.073 \pm 0.005$ \\
Val & $1.078 \pm 0.130$ & $0.498 \pm 0.026$ \\
Phe & $0.748 \pm 0.084$ & $0.366 \pm 0.023$ \\
Ile & $0.932 \pm 0.117$ & $0.391 \pm 0.016$ \\
Leu & $1.021 \pm 0.116$ & $0.489 \pm 0.026$ \\
Sum of essential amino acids & $6.416 \pm 0.815$ & $2.585 \pm 0.167$ \\
Asp & $0.973 \pm 0.105$ & $0.381 \pm 0.037$ \\
Glu & $1.308 \pm 0.173$ & $0.552 \pm 0.050$ \\
Ser & $0.485 \pm 0.080$ & $0.175 \pm 0.016$ \\
Gly & $0.484 \pm 0.089$ & $0.200 \pm 0.024$ \\
Ala & $0.723 \pm 0.078$ & $0.309 \pm 0.025$ \\
Tyr & $0.788 \pm 0.088$ & $0.186 \pm 0.009$ \\
Sum of total amino acids & $11.176 \pm 1.374$ & $4.388 \pm 0.324$ \\
\hline
\end{tabular}

accordingly enhance the production of subitaneously developing offspring in crowded $D$. pulex, suggesting that specific dietary amino acids are involved in triggering the switch between reproductive modes in the freshwater keystone herbivore Daphnia.

In eukaryotes, amino acids are required for a multitude of different physiological processes, such as muscle generation, synthesis of enzymes and peptide hormones, precursors of neurotransmitters or vitellogenesis (e.g. Harrison 1990; Lafont 2000). In principle, it is assumed that some amino acids are essential across the animal kingdom, i.e. that vertebrates and arthropods presumably share the same requirements for dietary amino acids (Cowey and Forster 1971; Peters and De Bernardi 1987; Guisande et al. 1999). It has been shown that the availability of dietary amino acids significantly affects the reproduction of terrestrial insects, such as butterflies (Bauerfeind and Fischer 2005; Mevi-Schütz and Erhardt 2005) or the cyclical parthenogenetic aphids (e.g. Dixon et al. 1993; Simpson et al. 1995). It has also been suggested that dietary amino acids promote high fecundity in marine copepods (Kleppel et al. 1998; Guisande et al. 1999, 2000; Helland et al. 2003). As yet, however, the nutritional requirements of the freshwater herbivore Daphnia for single amino acids have not been studied.

Our supplementation experiments reveal that the amino acids arginine and histidine are of particular importance for Daphnia. In a decapod crustacean, arginine and histidine were the principal free amino acids detected during embryogenesis (Rosa et al. 2005) and, in a juvenile marine shrimp species, a certain dietary arginine level was determined that supports optimal growth (Chen et al. 1992). Moreover, it has been reported that egg production in a marine copepod is significantly related to the arginine and histidine content of its diet (Kleppel et al. 1998). A potential arginine limitation of marine copepods was suggested by Anderson et al. (2004) based on comparisons between amino acid profiles of consumers (copepods) and their food (seston). Together with the finding that the free amino acid pool of Daphnia magna is dominated by arginine, histidine, alanine and glutamic acid (Gardener and Miller 1981), these examples imply that arginine and histidine are important nutrients which play significant roles in the metabolism of crustaceans. A high metabolic requirement for arginine and histidine in crustaceans would also explain why the low arginine and histidine content of $S$. obliquus influenced the reproductive mode and the neonate production of Daphnia in our experiments. However, additional amino acids also seem to be important, at least when supplied in combination with arginine and histidine. For example, the threshold concentration of arginine and histidine for suppressing ephippia production was $6.25 \mu \mathrm{M}$ when supplied in combination with eight other amino acids (Fig. 2), but $25 \mu \mathrm{M}$ were not sufficient to completely stop ephippia production when arginine and histidine were supplied individually (Fig. 4). These results suggest that dietary amino acids provoke complex synergistic effects on Daphnia reproduction ands may support the assumption that these amino acids are essential and not substitutable, 

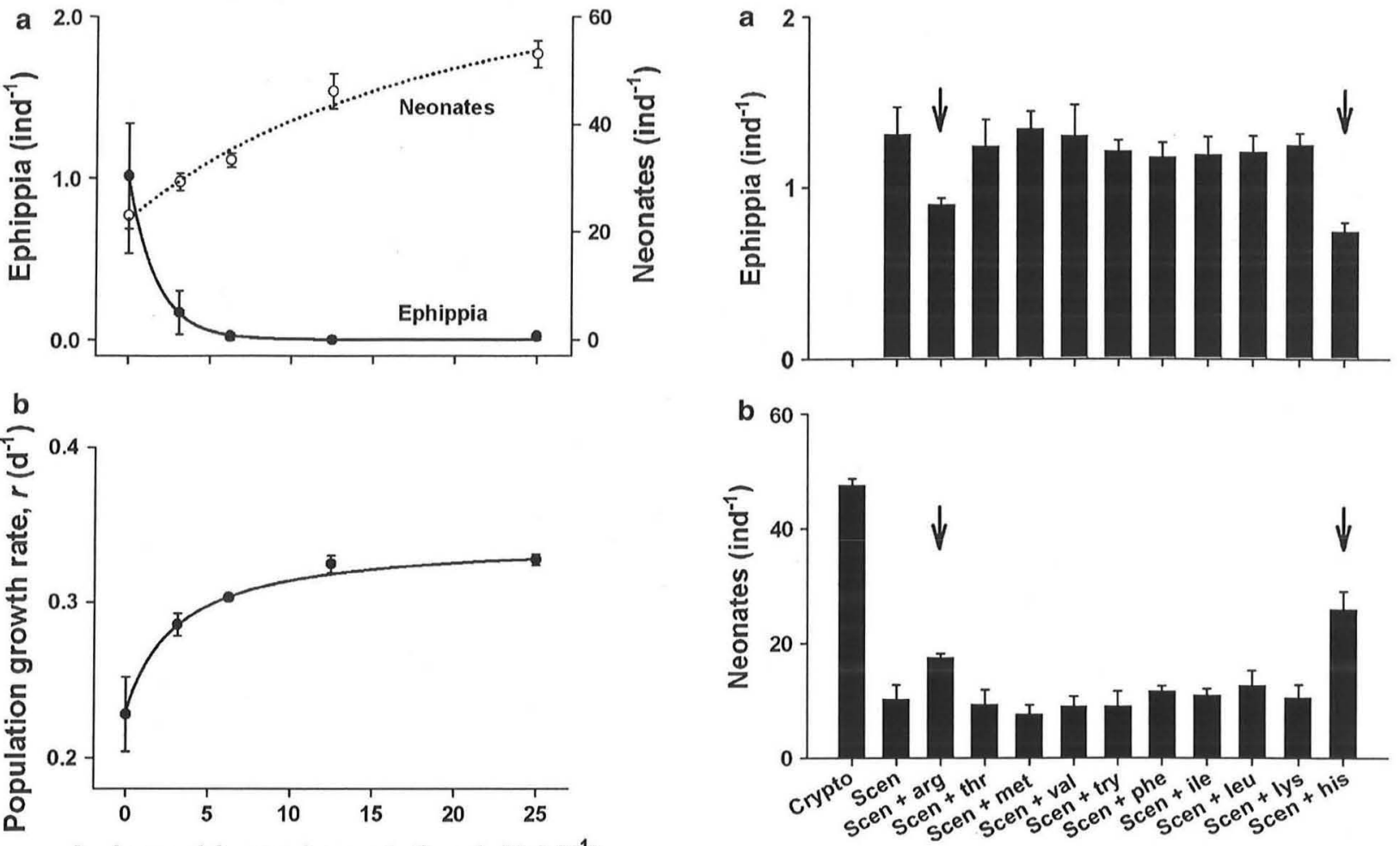

Fig. 2 Cumulative numbers (mean \pm SE) of ephippia and neonates produced per individual (a) and population growth rates $(r)(\mathbf{b})$ within 16 days of $D$. pulex being fed $S$. obliquus and supplemented with increasing amounts of an amino acid mixture containing ten different amino acids, leading to concentrations of $0-25 \mu \mathrm{M} \mathrm{AA}^{-1}$

as supplementation of only one amino acid may lead to a deficiency in a different essential amino acid.

In our experiments two scenarios of amino acid up-take by daphnids can be considered: (1) directly from the surrounding water, or (2) via the ingestion of food algae that may have concentrated amino acids at their surface by adsorption or in their cells by the direct uptake of dissolved amino acids. A direct uptake of the amino acid leucine by S. obliquus was described by Kamjunke and Tittel (2008). To the contrary, it has been demonstrated that nauplii of Artemia fransiscana are able to take up dissolved free amino acids directly from the surrounding water, even though the uptake of particle-bound amino acids has been shown to be more efficient (Tonheim et al. 2000). This latter result is in accordance with our data which show that amino acids associated with Cryptomonas sp. were much more efficient in reducing ephippia production than dissolved free amino acids supplemented to the water.

During a single molt cycle daphnids can produce either one ephippium or one clutch of several neonates. Furthermore, the production of an ephippium delays the

Fig. 3 Cumulative numbers of ephippia (a) and neonates produced per individual (b) within 16 days of $D$. pulex being fed Cryptomonas sp. (Crypto), S. obliquus (Scen), or S. obliquus supplemented with ten different amino acids (concentration $25 \mu \mathrm{M} \mathrm{AA}^{-1}$; see "Materials and methods" for AA abbreviations). Error bars Mean + SE. Arrows indicate the most effective amino acids, i.e. minimum ephippia production and maximum neonate production

production of subsequent clutches. The costs of producing a single ephippium have been estimated by Lynch (1983) to be equivalent to the cost of producing nine neonates; these calculations were based on dry mass differences between neonates and one ephippium of $D$. pulex. Our data from the regression analysis suggest a cost of $11.2 \pm 3.2$ neonates, respectively, a growth rate reduction of $0.07 \pm$ $0.006 \mathrm{day}^{-1}$ per ephippium produced. Consequently, the reduction of ephippia production due to amino acid supplementation resulted in a substantial enhancement of neonate production and the population growth rate of $D$. pulex. However, neonate production and population growth rate $(r)$ further increased after ephippia production had ceased, suggesting that food quality, i.e. the dietary amino acid content, affects the production of offspring in a manner independent of the switch between reproductive modes. Following the switch between reproductive modes, population growth rates and neonate production further increased by 8 and $60 \%$, respectively, when amino acid supplementation was enhanced from 6.25 to $25 \mu \mathrm{M} \mathrm{AA}^{-1}$. The effect of amino acid supplementation on neonate 

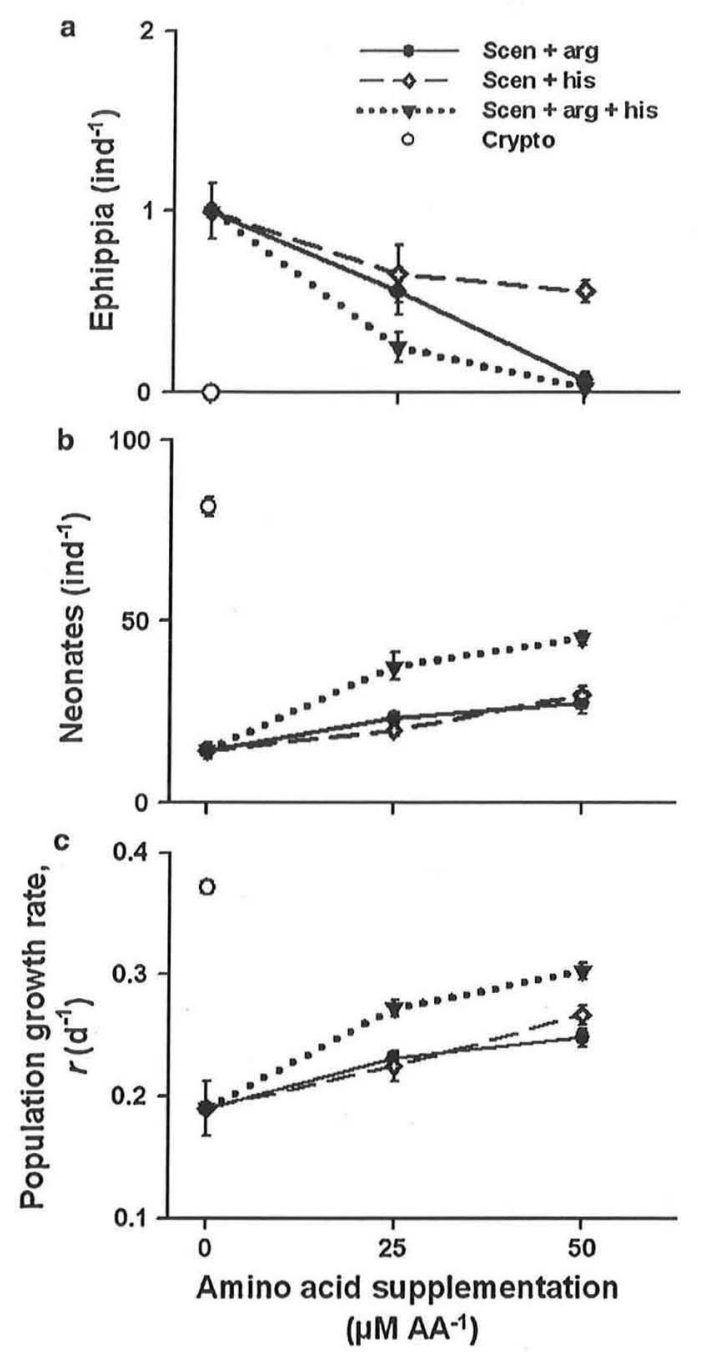

Fig. 4 Cumulative numbers (means \pm SE) of ephippia (a), neonate production produced per individual (b) and population growth rates (r) (c) within 16 days of $D$. pulex being fed Cryptomonas sp. (Crypto), or S. obliquus (Scen) supplemented with 1, 25 and $50 \mu \mathrm{M}$ $\mathrm{AA}^{-1}$, respectively, of arginine (arg) and histidine (his) separately or combined

production was more pronounced than that on population growth rates because neonate numbers showed the largest increase in the third clutch, which affects the calculation of population growth rates using the Euler-Lotka equation less than earlier clutches.

It has been reported that the comparatively poor food quality of S. obliquus for Daphnia spp. is due to a deficiency in essential long chain PUFAs (Weers and Gulati 1997; Von Elert 2002; Brzeziński and Von Elert 2007). Taking Liebig's law of the minimum into consideration, this explanation appears to be incompatible with our data, which imply that the performance of daphnids feeding on $S$. obliquus is constrained by a deficiency in specific amino acids. Recently, however, it has been shown that the growth and reproduction of the herbivore Daphnia can be limited by more than one nutrient simultaneously (MartinCreuzburg et al. 2009), which suggests a colimitation of Daphnia spp. feeding on S. obliquus by specific fatty acids and amino acids.

In nature, a major factor determining the growth and reproduction of Daphnia is the composition of its food, which undergoes pronounced seasonal changes due to the succession of algal species and seasonally varying ratios of algal to detritus biomass (Sommer 1985). We used crowding conditions and high food quantities in our experimental study to enhance ephippia production. Thus, our experimental set-up represents a scenario with high Daphnia abundance and high food availability, which occurs in nature at least on a seasonal basis. In Lake Constance, the start of ephippia production in early summer is just after the peak of the spring algae bloom (Jankowski and Straile 2004), which is a time characterised by strong changes in phytoplankton composition (Sommer 1985) and, consequently, in food quality for Daphnia (Wacker and Von Elert 2001); however, food concentrations are still high, and there is, therefore, an energy supply for resting egg production. Abrusán et al. (2007) reported that, at strongly limiting food concentrations, resting egg production in D. pulicaria is potentially constrained by a dietary deficiency in essential PUFAs, which suggests that food quality, in terms of PUFAs, in conjunction with environmental stimuli indicating deteriorating growth conditions affect the induction of resting egg production. In contrast, we show here that the presence of single dietary amino acids can suppress environmentally induced ephippia production at high food concentrations. Thus, the start of ephippia production in Daphnia and the number of mothers switching to resting egg production in nature are apparently a result of a complex interplay between inducing and suppressing environmental cues. Further studies are required to assess the relative significance of single cues in triggering the switch between reproductive modes under field conditions.

In conclusion, our results demonstrate that the growth and reproduction of $D$. pulex is significantly affected by the availability of dietary amino acids, particularly by arginine and histidine. We show that these amino acids are involved in triggering the switch between reproductive modes, i.e. they suppress resting egg production in crowded Daphnia and accordingly enhance parthenogenetic fecundity. The effect of amino acids on ephippia production differs qualitatively from that of fatty acids as the latter do not affect the switch between reproductive modes, but they are an important resource for the production of resting eggs (Abrusán et al. 2007; Koch et al. 2009). In our experiments, we used an obligate parthenogenetic D. pulex clone. Obligate parthenogenetic Daphnia clones are common in 
Table 2 Results of the analysis of covariance (ANCOVA) with the supplemented concentration ( 25 or $50 \mu \mathrm{M})$ of amino acid as a covariate and the specific amino acid (argine, histidine, or argine and histidine) as a categorical factor

\begin{tabular}{lll}
\hline Covariate/categorical factor & Model & Significance \\
\hline Ephippia production & $\left(\right.$ Model $\left.R^{2}=0.61, p<0.001\right)$ & $p=0.004$ \\
AA concentration & $F_{1,20}=10.43$ & $p<0.001$ \\
AA treatment & $F_{2,20}=10.78$ & $\left(\right.$ Model $\left.R^{2}=0.78, p<0.001\right)$ \\
Neonate production & $F_{1,20}=12.78$ \\
AA concentration & $F_{2,20}=29.45$ \\
AA treatment & $\left(\right.$ Model $\left.R^{2}=0.75, p<0.001\right)$ \\
Population growth rate & $F_{1,20}=19.80$ \\
AA concentration & $F_{2,20}=20.04$ \\
AA treatment &
\end{tabular}

$A A$, Amino acid

No significant interaction effects (all $p>0.1$ ) were detected for ephippia and neonate production or for population growth rate

natural systems and often coexist with sexual clones of the same species (Innes and Singleton 2000; Innes et al. 2000; Omilian et al. 2006). In an earlier study, we demonstrated that protein supplementation can suppress ephippia production in the asexual D. pulex clone used here, as well as in a sexual $D$. galeata clone (Koch et al. 2009), suggesting that amino acids are also involved in triggering the switch between reproductive modes in sexual Daphnia clones. However, further studies are necessary to determine potential clone- and/or species-specific differences in resting egg production in response to dietary amino acid availability.

Hence, in addition to minerals (Sterner and Elser 2002), PUFAs (Müller-Navarra et al. 2000; Wacker and Von Elert 2001; Von Elert 2002) and sterols (Von Elert et al. 2003; Martin-Creuzburg et al. 2005, 2009), dietary amino acids have to be considered as potentially limiting resources which constrain the performance of Daphnia. Our data imply that the availability of single dietary amino acids may affect seasonal dynamics, long-term persistence, dispersal in and among ecosystems and, consequently, the genetic diversity of Daphnia populations in the field.

Acknowledgments We thank C. Koppe for amino acid analyses. C. Gebauer, P. Merkel and S. Ballert provided technical and C. Kolb and F. von Netzer provided experimental assistance. We also thank K.-O. Rothhaupt, A. Wacker and S. Raub for helpful comments on an earlier draft of this manuscript. The project was partially funded by the 'Umwelt und Wohnen' foundation, the Leibniz foundation and the University of Konstanz.

\section{References}

Abrusán G, Fink P, Lampert W (2007) Biochemical limitation of resting egg production in Daphnia. Limnol Oceanogr 52:1724-1728

Alekseev V, Lampert W (2001) Maternal control of resting-egg production in Daphnia. Nature 414:899-901
Anderson TR, Boersma M, Raubenheimer D (2004) Stoichiometry: linking elements to biochemicals. Ecology 85:1193-1202

Bauerfeind SS, Fischer K (2005) Effects of adult-derived carbohydrates, amino-acids and micronutrients on female reproduction in a fruit-feeding butterfly. J Insect Physiol 51:545-554

Berner D, Blanckenhorn WU, Korner C (2005) Grasshoppers cope with low host plant quality by compensatory feeding and food selection: N limitation challenged. Oikos 111:525-533

Brzeziński T, Von Elert E (2007) Biochemical food quality effects on a Daphnia hybrid complex. Limnol Oceanogr 52:2350-2357

Bukovinszky T, Poelman EH, Gols R, Prekatsakis G, Vet LEM, Harvey JA, Dicke M (2009) Consequences of constitutive and induced variation in plant nutritional quality for immune defence of a herbivore against parasitism. Oecologia 160:299-308

Cáceres C (1998) Interspecific variation in the abundance, production, and emergence of Daphnia diapausing eggs. Ecology 79:16991710

Carpenter SR, Cole JJ, Hodgson JR, Kitchell JF, Pace ML, Bade D, Cottingham KL, Essington TE, Houser JN, Schindler DE (2001) Trophic cascades, nutrients, and lake productivity: whole-lake experiments. Ecol Monogr 71:163-186

Chen H-Y, Leu Y-T, Roelants I (1992) Quantification of arginine requirements of juvenile marine shrimp, Penaeus monodon, using microencapsulated arginine. Mar Biol 114:229-233

Cowey CB, Forster JRM (1971) The essential amino-acid requirements of the prawn Palaemon serratus. The growth of prawns on diets containing proteins of different amino acid compositions. Mar Biol 10:77-81

Dixon AFG, Wellings PW, Carter C, Nichols JFA (1993) The role of food quality and competition in shaping the seasonal cycle in the reproductive activity of the sycamore aphid. Oecologia 95:89-92

Gardener WS, Miller WH (1981) Intracellular composition and net release rates of free amino acids in Daphnia magna. Can J Fish Aquat Sci 38:157-162

Grossart H-P, Engel A, Arnosti C, De La Rocha CL, Murray AE, Passow U (2007) Microbial dynamics in autotrophic and heterotrophic seawater mesocosms. III. Organic matter fluxes. Aquat Microb Ecol 49:143-156

Guillard RR (1975) Cultures of phytoplankton for feeding of marine invertebrates. In: Smith WL, Chanley MH (eds) Culture of marine invertebrate animals. Plenum, New York, pp 29-60

Guisande C, Maneiro I, Riveiro I (1999) Homeostasis in the essential amino acid composition of the marine copepod Euterpina acutifrons. Limnol Oceanogr 44:691-696 
Guisande C, Riveiro I, Maneiro I (2000) Comparisons among the amino acid composition of females, eggs and food to determine the relative importance of food quantity and food quality to copepod reproduction. Mar Ecol Prog Ser 202:135-142

Hairston NG Jr (1996) Zooplankton egg banks as biotic reservoirs in changing environments. Limnol Oceanogr 41:1087-1092

Hairston NG Jr, Kearns CM (2002) Temporal dispersal: ecological and evolutionary aspects of zooplankton egg banks and the role of sediment mixing. Integ Comp Biol 42:481-491

Harrison K (1990) The role of nutrition in maturation, reproduction and embryonic development of decapod crustacea: a review. J Shellfish Res 9:1-28

Helland S, Nejstgaard JC, Humlen R, Fyhn HJ, Båmstedt U (2003) Effects of season and maternal food on Calanus finmarchicus reproduction, with emphasis on free amino acids. Mar Biol 142:1141-1151

Innes DJ, Singleton DR (2000) Variation in allocation to sexual and asexual reproduction among clones of cyclically parthenogenetic Daphnia pulex (Crustacea: Cladocera). Biol J Linn Soc 71:771-787

Innes DJ, Fox CJ, Winsor GL (2000) Avoiding the cost of males in obligately asexual Daphnia pulex (Leydig). Proc R Soc Lond B 267:991-997

Jankowski T, Straile D (2004) Allochronic differentiation among Daphnia species, hybrids and backcrosses: the importance of sexual reproduction for population dynamics and genetic architecture. J Evol Biol 17:312-321

Jüttner F, Leonhardt J, Möhren S (1983) Environmental factors affecting the formation of mesityloxide, dimethylallylic alcohol and other volatile compounds excreted by Anabaena cylindrica. J Gen Micrbiol 129:407-412

Kamjunke N, Tittel J (2008) Utilization of leucine by several phytoplankton species. Limnologica 38:360-366

Kazana E, Pope TW, Tibbles L, Bridges M, Pickett JA, Bones AM, Powell G, Rossiter JT (2007) The cabbage aphid: a walking mustard oil bomb. Proc Biol Sci 274:2271-2277

Kleiven OT, Larsson P, Hobæk A (1992) Sexual reproduction in Daphnia magna requires three stimuli. Oikos 65:197-206

Kleppel GS, Burkhart CA, Houchin L (1998) Nutrition and regulation of egg production in the calanoid copepode Acartia tonsa. Limnol Oceanogr 43:1000-1007

Koch U, Von Elert E, Straile D (2009) Food quality triggers the reproductive mode in the cyclical parthenogen Daphnia (Cladocera). Oecologia 159:317-324

Lafont R (2000) The endocrinology of invertebrates. Ecotoxicology 9:41-57

Lampert W (1987) Feeding and nutrition in Daphnia. In: Peters RH, De Bernardi R (eds) Daphnia. Mem Ist Ital Idrobiol 45:143-192

Leibold MA (1989) Resource edibility and the effects of predators and productivity on the outcome of trophic interactions. Am Nat 134:922-949

Louette G, De Meester L (2005) High dispersal capacity of cladoceran zooplankton in newly founded communities. Ecology 86:353-359

Lynch M (1983) Ecological genetics of Daphnia pulex. Evolution $37: 358-374$

Martin-Creuzburg D, Wacker A, Von Elert E (2005) Life history consequences of sterol availability in the aquatic keystone species Daphnia. Oecologia 144:362-372

Martin-Creuzburg D, Sperfeld E, Wacker A (2009) Colimitation of a freshwater herbivore by sterols and polyunsaturated fatty acids. Proc R Soc B 276:1805-1814

Mevi-Schütz J, Erhardt A (2005) Amino acids in nectar enhance butterfly fecundity: a long-awaited link. Am Nat 165:411-419
Müller-Navarra DC, Brett MT, Liston AM, Goldman CR (2000) A highly unsaturated fatty acid predicts carbon transfer between primary producers and consumers. Nature 403:74-77

Omilian AR, Cristescu MEA, Dudycha JL, Lynch M (2006) Ameiotic recombination in asexual lineages of Daphnia. Proc Nat Acad Sci USA 103:18638-18643

Panov VE, Krylov PI, Riccardi N (2004) Role of diapause in dispersal and invasion success by aquatic invertebrates. J Limnol 63:56-69

Peters RH, De Bernardi R (1987) Composition and nutrition. In: Daphnia PetersRH, De Bernardi R (eds) Memorie dell'instituto di idrobiologia dott. Marco De Marchi, Milan, pp 201-203

Rosa R, Calado R, Andrade AM, Narciso L, Nunes ML (2005) Changes in amino acids and lipids during embryogenesis of the European lobster, Homarus gammarus (Crustacea: Decapoda). Comp Biochem Physiol B 140:241-249

Schatz GS, McCauley E (2007) Foraging behavior by Daphnia in stoichiometric gradients of food quality. Oecologia 153:10211030

Schwartz SS, Hebert PD (1987) Methods for the activation of the resting eggs of Daphnia. Freshw Biol 117:373-379

Simpson SJ, Abisgold JD, Douglas AE (1995) Response of the pea aphid (Acyrthosiphon pisum) to variation in dietary levels of sugar and amino acids: the significance of dietary amino acids quality. J Insect Physiol 41:71-75

Ślusarczyk M (1995) Predator-induced diapause in Daphnia. Ecology 76:1008-1013

Ślusarczyk M (2001) Food threshold for diapause in Daphnia under the threat of fish predation. Ecology 82:1089-1096

Smilanich AM, Dyer LA, Chambers JQ, Bowers MD (2009) Immunological cost of chemical defence and the evolution of herbivore diet breadth. Ecol Lett 12:612-621

Sommer U (1985) Seasonal succession of phytoplankton in lake Constance. Bioscience 35:351-357

Sterner RW, Elser JJ (2002) Ecological stoichiometry: the biology of elements from molecules to the biosphere. Princeton University Press, Princeton

Stross RG, Hill JC (1965) Diapause induction in Daphnia requires two stimuli. Science 150:1462-1464

Tonheim SK, Woven W, Rønnestad I (2000) Enrichment of Artemia with free methionine. Aquaculture 190:223-235

Twombly S, Clancy N, Burns CW (1998) Life history consequences of food quality in the freshwater copepod Boeckella triarticulata. Ecology 79:1711-1724

Von Elert E (2002) Determination of limiting polyunsaturated fatty acids in Daphnia galeata using a new method to enrich food algae with single fatty acids. Limnol Oceanogr 47:1764-1773

Von Elert E, Martin-Creuzburg D, Le Coz JR (2003) Absence of sterols constrains carbon transfer between cyanobacteria and a freshwater herbivore (Daphnia galeata). Proc R Soc B 270:1209-1214

Wacker A, Von Elert E (2001) Polyunsaturated fatty acids: evidence for non-substitutable biochemical resources in Daphnia galeata. Ecology 82:2507-2520

Weers PMM, Gulati RD (1997) Growth and reproduction of Daphnia galeata in response to changes in fatty acids, phosphorus, and nitrogen in Chlamydomonas reinhardtii. Limnol Oceanogr 42:1584-1589

Weider LJ, Jeyasingh PD, Looper KG (2008) Stoichiometric differences in food quality: impacts on genetic diversity and the coexistence of aquatic herbivores in a Daphnia hybrid complex. Oecologia 158:47-55 